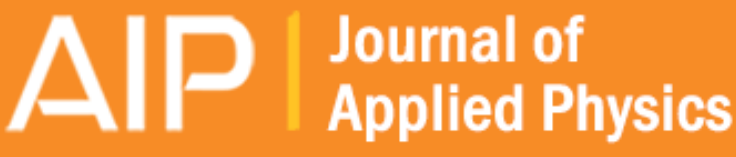

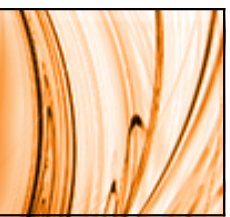

Indium tin oxide nanowires as hyperbolic metamaterials for near-field radiative heat

\section{transfer}

Jui-Yung Chang, Soumyadipta Basu, and Liping Wang

Citation: Journal of Applied Physics 117, 054309 (2015); doi: 10.1063/1.4907581

View online: http://dx.doi.org/10.1063/1.4907581

View Table of Contents: http://scitation.aip.org/content/aip/journal/jap/117/5?ver=pdfcov

Published by the AIP Publishing

\section{Articles you may be interested in}

Near-field radiative heat transfer between doped silicon nanowire arrays

Appl. Phys. Lett. 102, 053101 (2013); 10.1063/1.4790143

Near-field radiative heat transfer between chiral metamaterials

J. Appl. Phys. 112, 084309 (2012); 10.1063/1.4759055

Penetration depth in near-field radiative heat transfer between metamaterials

Appl. Phys. Lett. 99, 143107 (2011); 10.1063/1.3646466

Maximum near-field radiative heat transfer between thin films

Appl. Phys. Lett. 98, 243120 (2011); 10.1063/1.3600649

Maximum energy transfer in near-field thermal radiation at nanometer distances

J. Appl. Phys. 105, 093535 (2009); 10.1063/1.3125453

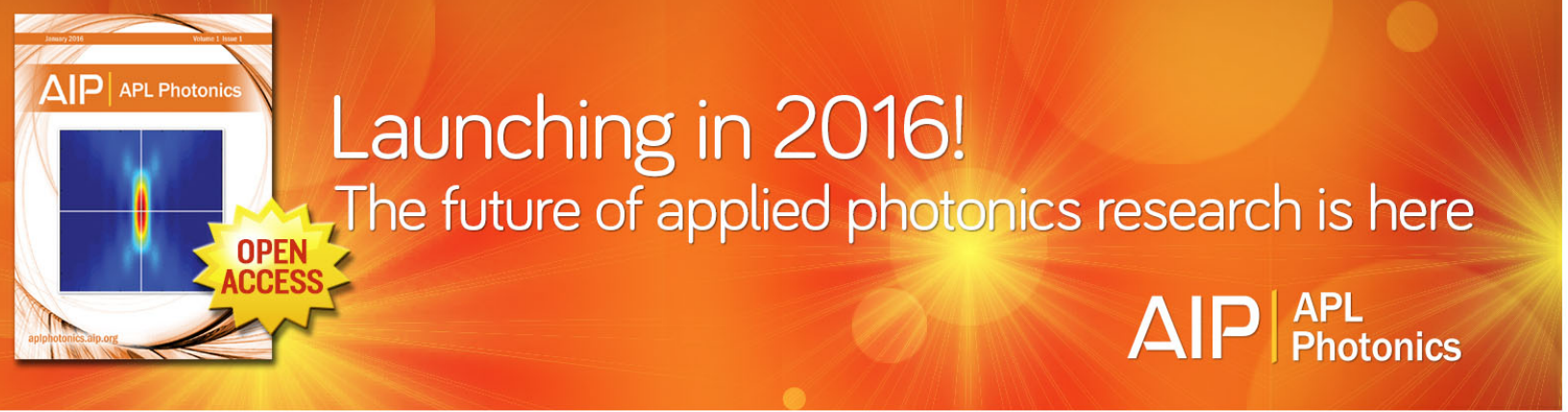




\title{
Indium tin oxide nanowires as hyperbolic metamaterials for near-field radiative heat transfer
}

\author{
Jui-Yung Chang, Soumyadipta Basu, ${ }^{\text {a) }}$ and Liping Wang ${ }^{\text {b) }}$ \\ School for Engineering of Matter, Transport, and Energy, Arizona State University, Tempe, Arizona 85287, \\ USA
}

(Received 24 September 2014; accepted 23 January 2015; published online 5 February 2015)

\begin{abstract}
We investigate near-field radiative heat transfer between Indium Tin Oxide (ITO) nanowire arrays which behave as type 1 and 2 hyperbolic metamaterials. Using spatial dispersion dependent effective medium theory to model the dielectric function of the nanowires, the impact of filling fraction on the heat transfer is analyzed. Depending on the filling fraction, it is possible to achieve both types of hyperbolic modes. At $150 \mathrm{~nm}$ vacuum gap, the heat transfer between the nanowires with 0.5 filling fraction can be 11 times higher than that between two bulk ITOs. For vacuum gaps less than $150 \mathrm{~nm}$ the heat transfer increases as the filling fraction decreases. Results obtained from this study will facilitate applications of ITO nanowires as hyperbolic metamaterials for energy systems. (C) 2015 AIP Publishing LLC. [http://dx.doi.org/10.1063/1.4907581]
\end{abstract}

\section{INTRODUCTION}

Radiative heat transfer between two media in the nearfield regime can exceed the blackbody heat transfer by orders of magnitude when surface waves are excited. The enhanced energy transfer can be utilized in energy systems, radiative cooling, thermal switches as well as rectifiers. ${ }^{1-8}$ Several studies have been performed in order to enhance the nearfield heat flux due to coupled surface modes in terms of different materials as well as structures. ${ }^{9-13}$ However, surfaces modes can be excited only within a narrow frequency band thereby precluding the possibility of wide-band enhancement of near-field heat flux. Recently, near-field heat transfer between hyperbolic metamaterials (HMMs) has attracted a lot of attention due to the possibility of exciting resonance-free hyperbolic modes over a large frequency regime by careful tuning of geometry and material properties.

Hyperbolic metamaterial is a kind of metamaterial based on different signs of dielectric functions in uniaxial medium. ${ }^{14}$ With opposite signs, instead of an ellipsoid where dielectric function components have same signs, an HMM forms a hyperboloid on the isofrequency plot that provides unlimited length of wave vector (high-k mode). ${ }^{15}$ This high-k mode allows evanescent waves to propagate inside HMMs instead of decaying away exponentially which makes it beneficial in several applications such as thermophotovoltaics ${ }^{16}$ and hyperlens. ${ }^{17}$ Furthermore, the enhancement due to the broadband singularity in photonic density of state (PDOS) which is supported by the high-k mode is also an important feature of HMMs that reveals its potential. ${ }^{18}$ HMMs can be classified by the signs of dielectric function components: type $1\left(\varepsilon_{\mathrm{O}, \text { eff }}>0, \varepsilon_{\mathrm{E}, \text { eff }}<0\right)$ and type $2\left(\varepsilon_{0, \text { eff }}<0, \varepsilon_{\mathrm{E}, \text { eff }}>0\right)$ HMMs. These two special sets of material property can be achieved by metaldielectric constructed multilayer structures or metallic nanowire arrays embedded in dielectric hosts. HMMs can

\footnotetext{
a)soumya.005@gmail.com

b)liping.wang@asu.edu
}

be used in nano-imaging, sensing, Dyakonov plasmonics, fluorescence engineering, and thermal emission control within the range of visible and near-IR. ${ }^{19}$ Among them, thermal emission control is a very important concept in energy harvesting. HMMs can also enhance the performance of solar cells due to their broadband absorption. ${ }^{20}$ Additionally, nanowire HMMs can also enhance the nearfield heat transfer over that between bulks by orders of magnitude. ${ }^{21-23}$

In this study, we investigate the application of IndiumTin-Oxide (ITO) nanowires as hyperbolic metamaterials for near-field thermal radiation. Previous studies have looked into ITO thin films as plasmonic materials as well as transparent electrodes in solar cells and flat panel displays. ${ }^{24-27}$ Basu and Wang demonstrated that the near-field heat flux between doped silicon nanowire arrays can exceed that between two bulk doped Si media. ${ }^{11}$ Liu and Zhang have calculated the near-field heat flux between CNTs as HMMs with demonstrated higher heat transfer than that between graphite and silicon carbide. ${ }^{13}$ In this study, we have considered the spatial dispersion effect in the effective medium theory for nanowire HMMs for more accurate calculation of near-field radiative transfer.

Let us consider near-field radiative heat transfer between ITO nanowire arrays (ITONWAs) separated by vacuum gap of thickness $d$ as shown in Fig. 1. One of the nanowire arrays or the emitter (medium 1) is at temperature $T_{1}$ while the other ITONWA or the receiver (medium 2) is at temperature $T_{2}$. The two arrays are separated by a vacuum gap (medium 0 ) and are assumed to be in local thermal equilibrium. The wires are long enough for both the emitter and receiver to be considered semi-infinite. As a symmetric case, the filling fraction $f$ is assumed to be the same for both nanowire arrays. In previous studies, the nanowire arrays with large filling fractions can be considered as a homogenous medium with anisotropic optical properties by applying effective medium theory (EMT) ${ }^{11}$ However, since the spatial dispersion effect should not be excluded for nanowire structures with small filling fractions, ${ }^{28,29}$ the effective medium theory considering 


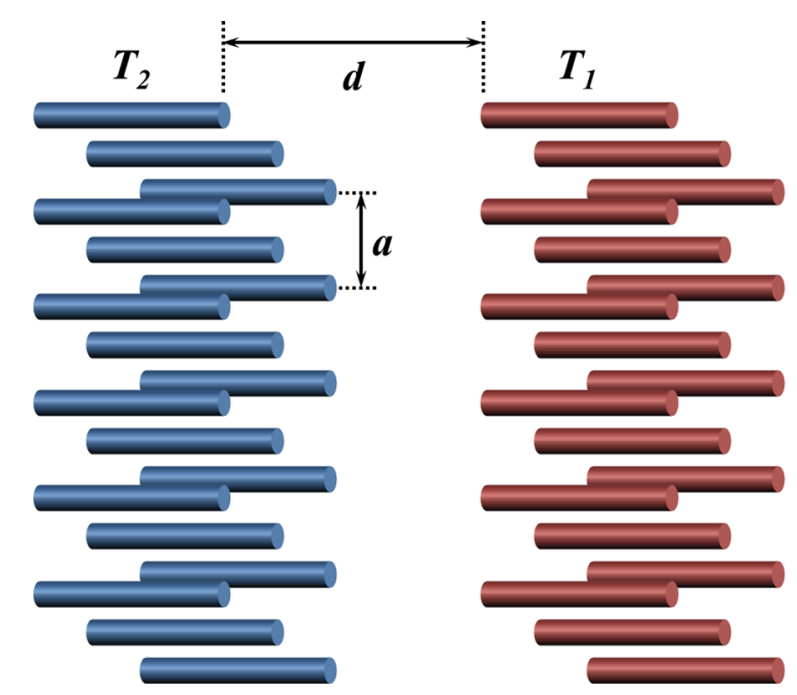

FIG. 1. Schematic of near-field radiation between ITONW arrays at different temperatures separated by a vacuum gap of width $d$.

spatial dispersion is applied here to describe the uniaxial optical properties of nanowire arrays. ${ }^{16,30}$

\section{THEORY}

In this study, the spatial dispersion dependent EMT is used to treat the ITONWA as homogenous layers since it accurately predicts optical properties for highly conductive nanorods with filling fractions less than $0.5 .{ }^{16,30}$ As part of the formulation for the ITONWA, vacuum is considered as the host medium and the ITO nanowires are taken as spatially separated embedded grains. For ordinary waves in which the electric field is perpendicular to the axis of nanowires, the effective dielectric function $\varepsilon_{\mathrm{O}, \text { eff }}$ is given as

$$
\varepsilon_{\mathrm{O}, \mathrm{eff}}=1+\frac{2}{\frac{1}{f} \frac{\varepsilon_{\mathrm{ITO}}+1}{\varepsilon_{\mathrm{ITO}}-1}-1} .
$$

Note that, Eq. (1) yields the same equation for ordinary waves given by Maxwell-Garnett EMT ${ }^{31}$ since the effect of spatial dispersion applies only for extraordinary waves. On the other hand for extraordinary waves where the electric field is parallel to the axis of the nanowires, the effective dielectric function $\varepsilon_{\mathrm{E}, \mathrm{eff}}$ is given by

$$
\varepsilon_{\mathrm{E}, \mathrm{eff}}=1+\frac{1}{\frac{1}{\left(\varepsilon_{\mathrm{ITO}}-1\right) f}-\left(\frac{c \beta}{\omega_{\mathrm{p}}}\right)^{2}},
$$

where $c$ is the speed of light in vacuum, $\beta$ is the wave vector component normal to the wire direction, and $\omega_{\mathrm{p}}$ is 3.362 $\times 10^{15} \mathrm{rad} / \mathrm{s}$ which is the plasmon frequency of ITO. ${ }^{25}$ Note that, since the effect of spatial dispersion is considered, the extraordinary dielectric function is not only a function of angular frequency $\omega$ like ordinary dielectric function. It is a function of wave vector component $\beta$ as well. In the above equations, the dielectric function of ITO, $\varepsilon_{\text {ITO }}$, is given by the Drude model

$$
\varepsilon_{\mathrm{ITO}}=\varepsilon_{\infty}-\frac{\omega_{\mathrm{p}}^{2}}{\omega^{2}+i \omega \Gamma} .
$$

The values of $\varepsilon_{\infty}$ and $\Gamma$ are 3.8 and $1.68 \times 10^{14} \mathrm{rad} / \mathrm{s},{ }^{25}$ respectively. The metallic nature of ITO results in a very small penetration depth for thermal radiation and therefore ITONWAs can be considered as semi-infinite in this study. The total heat transfer between two ITONWAs is given by ${ }^{1,32}$

$$
q^{\prime \prime}{ }_{\text {net }}=\frac{1}{4 \pi^{2}} \int_{0}^{\infty} d \omega\left[\Theta\left(\omega, T_{2}\right)-\Theta\left(\omega, T_{1}\right)\right] \int_{0}^{\infty} \beta \xi(\omega, \beta) d \beta,
$$

where $\Theta(\omega, T)$ is the mean energy of a Planck oscillator at angular frequency $\omega$ and temperature $T$, while the energy transmission coefficient $\xi(\omega, \beta)$ is given by

$$
\xi_{\text {prop }}(\omega, \beta)=\frac{\left(1-\left|r_{01}^{s}\right|^{2}\right)\left(1-\left|r_{02}^{s}\right|^{2}\right)}{\left|1-r_{01}^{s} r_{02}^{s} e^{i 2 \gamma_{0} d}\right|^{2}}+\frac{\left(1-\left|r_{01}^{p}\right|^{2}\right)\left(1-\left|r_{02}^{p}\right|^{2}\right)}{\left|1-r_{01}^{p} r_{02}^{p} e^{i 2 \gamma_{0} d}\right|^{2}}
$$

and

$$
\begin{aligned}
\xi_{\text {evan }}(\omega, \beta)= & \frac{4 \operatorname{Im}\left(r_{01}^{s}\right) \operatorname{Im}\left(r_{02}^{s}\right) e^{-2 \operatorname{Im}\left(\gamma_{0}\right) d}}{\left|1-r_{01}^{s} r_{02}^{s} e^{i 2 \gamma_{0} d}\right|^{2}} \\
& +\frac{4 \operatorname{Im}\left(r_{01}^{p}\right) \operatorname{Im}\left(r_{02}^{p}\right) e^{-2 \operatorname{Im}\left(\gamma_{0}\right) d}}{\left|1-r_{01}^{p} r_{02}^{p} e^{i 2 \gamma_{0} d}\right|^{2}},
\end{aligned}
$$

where Fresnel reflection coefficients for $s$ and $p$ polarization are obtained from Ref. 11.

\section{RESULTS AND DISCUSSION}

Figure 2 shows the filling fraction effect on the real and imaginary parts of ordinary dielectric function. Clearly the epsilon-near-pole (ENP) enhancement mode, which is caused by the spike of the imaginary part of dielectric function, ${ }^{33}$ becomes stronger with increasing filling fraction since the spikes of imaginary part appear to be manifest with larger filling fraction as revealed in Fig. 2(b). Note that, since the real part of ordinary dielectric function is always positive with small filling fractions (i.e., $f=0.1$ and 0.2 ), the ITONWA cannot behave as type 2 HMM under these circumstances. In Fig. 3, both the filling fraction effect and the normalized $\beta$ effect on the real part of extraordinary dielectric function are presented. As the filling fraction decreases and $\beta$ increases, the region of $\operatorname{Re}\left(\varepsilon_{\mathrm{E}}\right)<0$ shifts towards lower frequency. Note that, the effect of spatial dispersion is negligible when $\beta$ is less than 1 (i.e., propagating waves), however, it should not be neglected for evanescent waves $(\beta>1)$ as demonstrated in Fig. 3(b).

Figure 4 is a contour plot of $\xi(\omega, \beta)$ for $\mathrm{p}$ polarized waves as a function of $\omega$ and $\beta$ for different filling fractions during near-field radiative heat transfer between two nanowire arrays separated by a $20 \mathrm{~nm}$ vacuum gap. The temperature of the emitter is set to $1500 \mathrm{~K}$ while the receiver is set to $0 \mathrm{~K}$. The enhancement of ITONW can be identified through looking into the property of ITONW. For $f=0.1$, depending on the normalized $\beta$, ITONW behaves as type 1 HMM and effective dielectric at lower and higher frequency, respectively. Thus, the broad band enhancement in transmission coefficient at lower frequency is due to hyperbolic behavior and the small enhancement around $1.5 \times 10^{15} \mathrm{rad} / \mathrm{s}$ is due to ENP. For $f=0.3$, the broad band enhancement at lower 
(a)

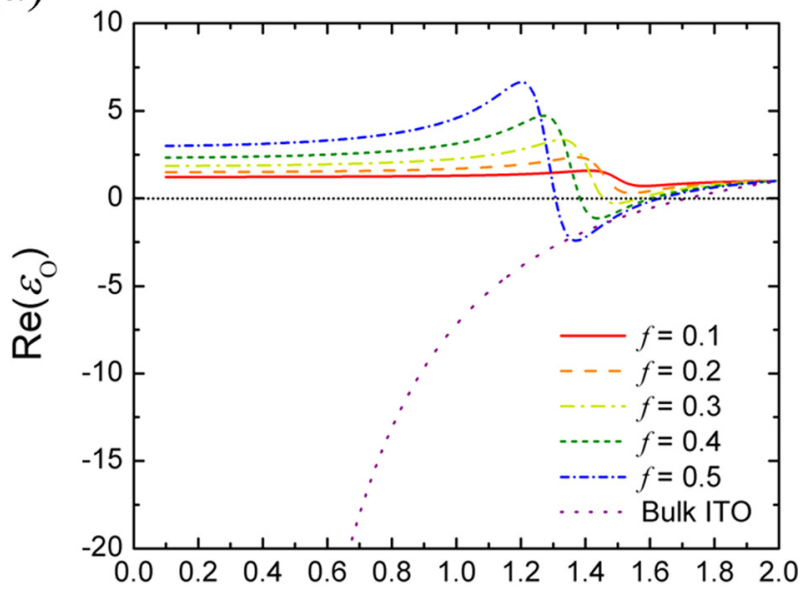

Angular Frequency, $\omega\left(\times 10^{15} \mathrm{rad} / \mathrm{s}\right)$

(b)

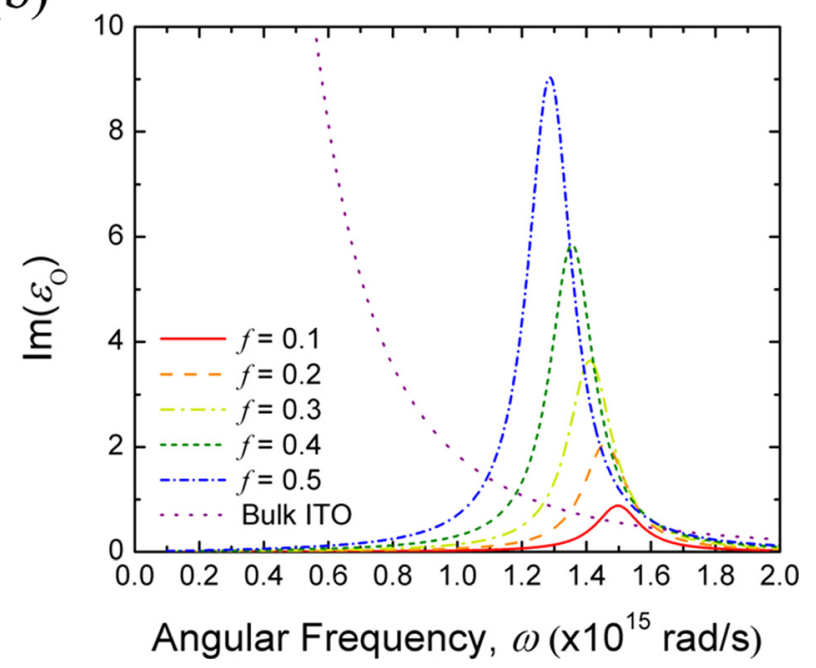

FIG. 2. Spectral plot of (a) real and (b) imaginary part of the ordinary dielectric function for an ITO nanowire array with different filling fractions.

frequency is also caused by type $1 \mathrm{HMM}$, but the region of type $1 \mathrm{HMM}$ shifts to higher frequency with increasing filling fraction. The strengthened enhancement around $1.5 \times 10^{15} \mathrm{rad} / \mathrm{s}$ is in fact due to type $2 \mathrm{HMM}$ which only occurs within a narrow band. For $f=0.5$, both the type 1 and type $2 \mathrm{HMM}$ enhancement modes occur similar to $f=0.3$. However, interestingly, the two enhancement bands form a single broadband enhancement region instead of two separate enhancement modes since the range of type 1 and type 2 HMM are right next to each other. This can be beneficial for some applications which requires continuous broadband enhancement in the field of energy conversion. In addition, the spectral location of ENP overlaps with that of type 2 HMM with both $f=0.3$ and 0.5 , and therefore cannot clearly be observed here since the enhancement strength of type 2 HMM mode is much stronger than that of ENP with p polarized waves. Note that, since HMM hyperbolic behavior occurs under all filling fractions, the strong near infrared resonance for ITONWAs irrespective of the filling fraction make them suitable candidates as emitter for near-field thermophotovoltaic devices. (a)

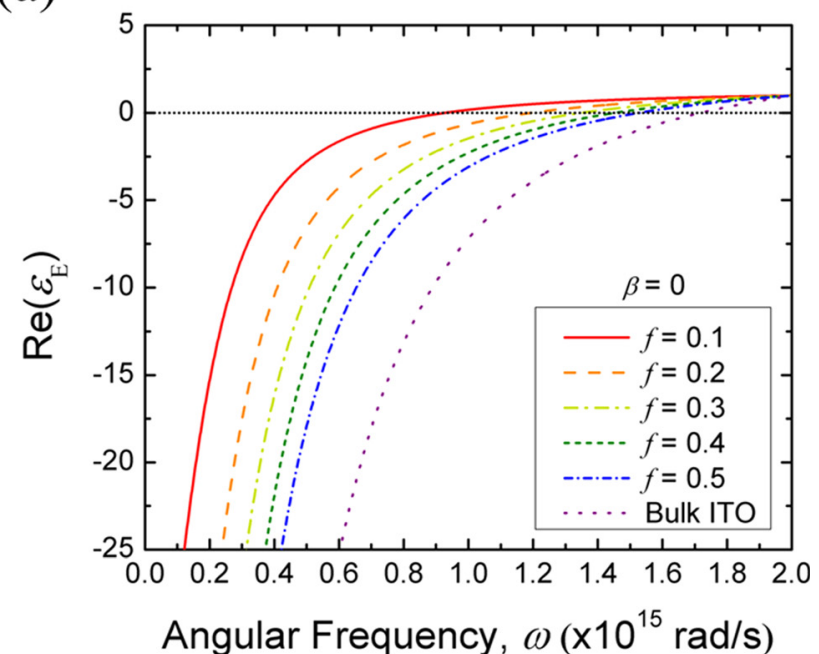

(b)

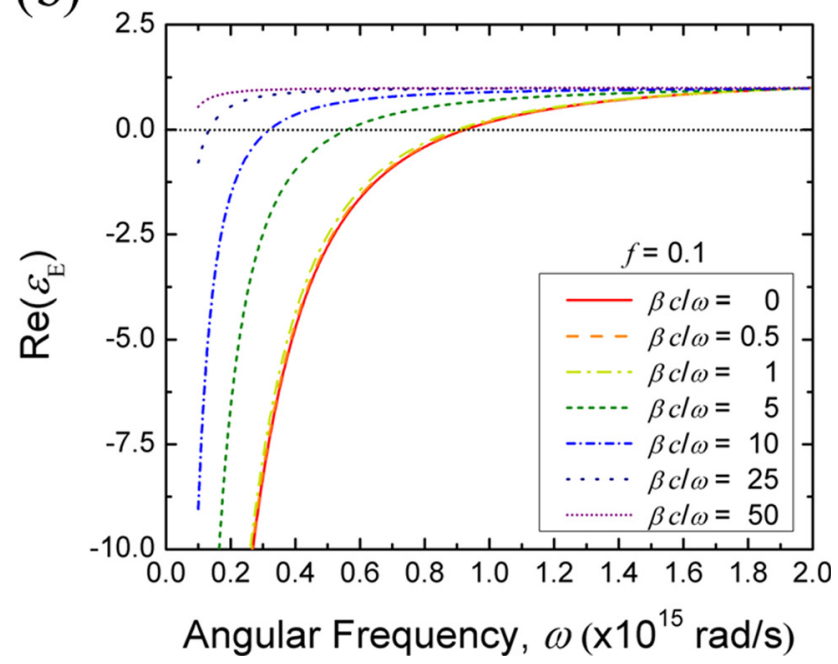

FIG. 3. Spectral plot of the real part of extraordinary dielectric function for an ITO nanowire array with different (a) filling fractions and (b) normalized wave vector component on $\mathrm{x}$ direction.

Figure 5 plots the total near-field heat transfer as a function of the vacuum gap separating the ITONWAs for different filling fractions. The expression for the total heat transfer is given in Eq. (4). The heat transfer for ITONWAs is normalized with respect to that between two bulk ITOs. The main enhancement between two bulk ITOs is due to coupled SPP whose strength increases as the vacuum gap decreases. Therefore, at very small gap distance such as $10 \mathrm{~nm}$, the heat flux between two ITONWAs is less than that between two bulk ITOs. However, with increasing vacuum gap to around $150 \mathrm{~nm}$, the gap distance effect on HMM hyperbolic modes is less than that on the coupling strength of SPP since HMM hyperbolic modes are due to the material property. Therefore, the heat flux between ITONWAs becomes nearly 11 times higher than that between bulk ITOs at $150 \mathrm{~nm}$ vacuum gap. Although further increment of gap distance will result in less enhancement due to the fast weakening of type 2 enhancement mode (to be shown by Fig. 7), the normalized heat flux still reaches about 7 at $d=1 \mu \mathrm{m}$. While Fig. 4 indicated that the near-field heat transfer at $20 \mathrm{~nm}$ vacuum gap 
(a)

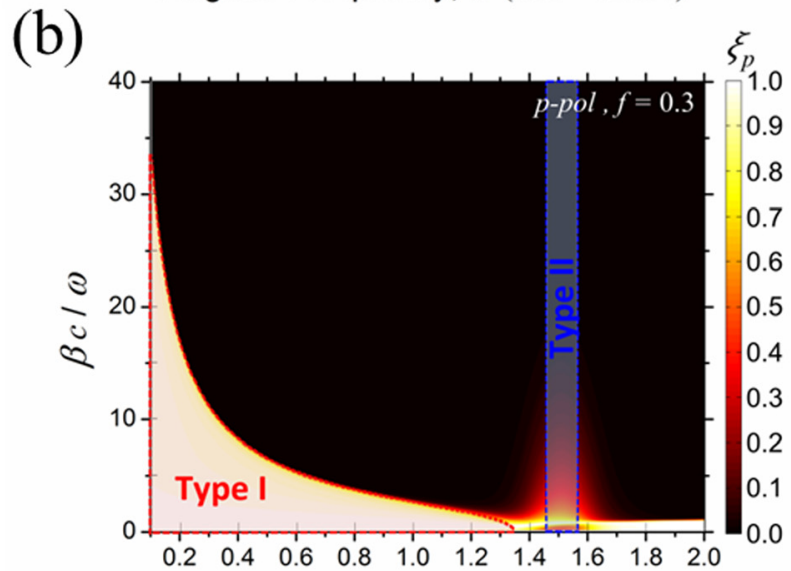

Angular Frequency, $\omega\left(\times 10^{15} \mathrm{rad} / \mathrm{s}\right)$

(c)

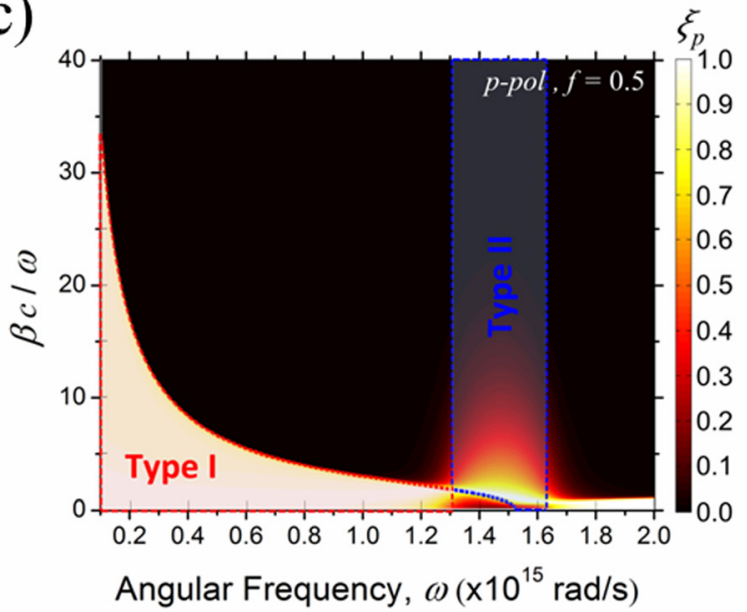

FIG. 4. Contour plot of $s(\beta, \omega)$ for different filling fractions: (a) 0.1, (b) 0.3, and (c) 0.5 at a vacuum gap of $20 \mathrm{~nm}$.

should increase as the filling fraction increases, Fig. 5 shows the same behavior only when $d$ is less than $150 \mathrm{~nm}$. Beyond $150 \mathrm{~nm}$, the trend changed and the total heat transfer is maximum for $f=0.3$ and minimum for $f=0.1$. This is also due to the fast weakening of type 2 enhancement mode which will result in the drop of overall heat flux on $f=0.4$ and 0.5 where type $2 \mathrm{HMM}$ enhancement is stronger. Figure 5 therefore shows the effect of vacuum gap on both HMM hyperbolic behavior (ITONWAs for different filling fractions) and SPP resonance (bulk ITOs). Interestingly, the total heat

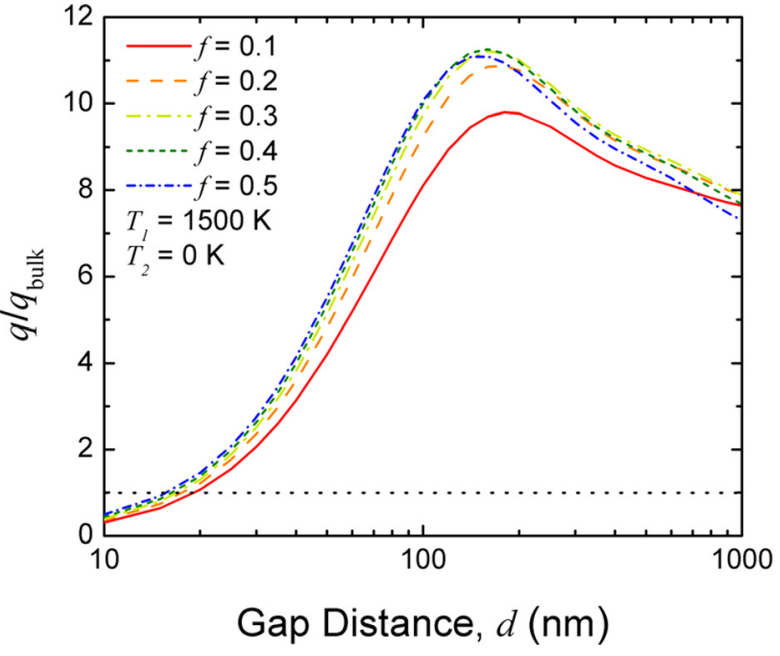

FIG. 5. Total heat flux between the ITONW arrays as a function of vacuum gap for different filling fractions. The heat flux is normalized with respect to that between two bulk ITOs.

transfer at $150 \mathrm{~nm}$ between ITONWAs with 0.5 filling fraction is more than one order of magnitude greater than that between semi-infinite ITO. On the other hand, for doped $\mathrm{Si}$ even for $f=0.5$, the heat transfer is only 2.5 times higher than that of bulk media at $150 \mathrm{~nm} .{ }^{11}$ ITONWs therefore provide a definite advantage in terms of enhancement in total heat transfer over doped semiconductors as $\mathrm{Si}$.

The spectral heat fluxes between two ITONWAs of $s$ and p polarized waves are shown in Figs. 6(a) and 6(b), respectively, for $20 \mathrm{~nm}$ vacuum gap. The spectral heat flux can be calculated by Eq. (4) without integrating over $\omega$. For convenience, the spectral heat flux between two semi-infinite bulk ITOs is also plotted in Fig. 6 along with different filling fractions for ITONWs. As shown in Fig. 6(a), there is no enhancement between semi-infinite bulk ITOs on s polarized waves at high frequency while the enhancement of ENP can clearly be seen for ITONWs. The shifting and strengthening of ENP enhancement agrees with the material property shown in Fig. 2. On the other hand, for $p$ polarized waves as presented in Fig. 6(b), there is one enhancement peak which corresponds to the excitation of SPP for semi-infinite ITO. For ITO nanowires, the peaks in spectral heat flux matches very well with the results from Fig. 4. In fact, both the shifting of type 1 hyperbolic mode and the broadening and strengthening of type 2 HMM can be observed with increasing filling fraction which the drop of spectral heat flux between two hyperbolic modes is also flattened. Note that, at this gap distance, the enhancement due to coupled SPP between two bulk ITOs is still stronger than that due to type 2 hyerbolic and ENP modes.

In Fig. 7, the spectral heat transfer between ITONWAs for different filling fractions is plotted at $d=200 \mathrm{~nm}$ in order to further explain the effect of gap distance on the overall heat transfer when vacuum gap is greater than $150 \mathrm{~nm}$. Again Figs. 7(a) and 7(b) are, respectively, for spectral plots of $\mathrm{s}$ and $\mathrm{p}$ polarized waves. As revealed by comparing Figs. 6(a) and 7(a), the spectral locations of ENP enhancement mode does not change with vacuum gap, but the strength is weaker. Similarly, the enhancement of type 1 hyperbolic 
(a)

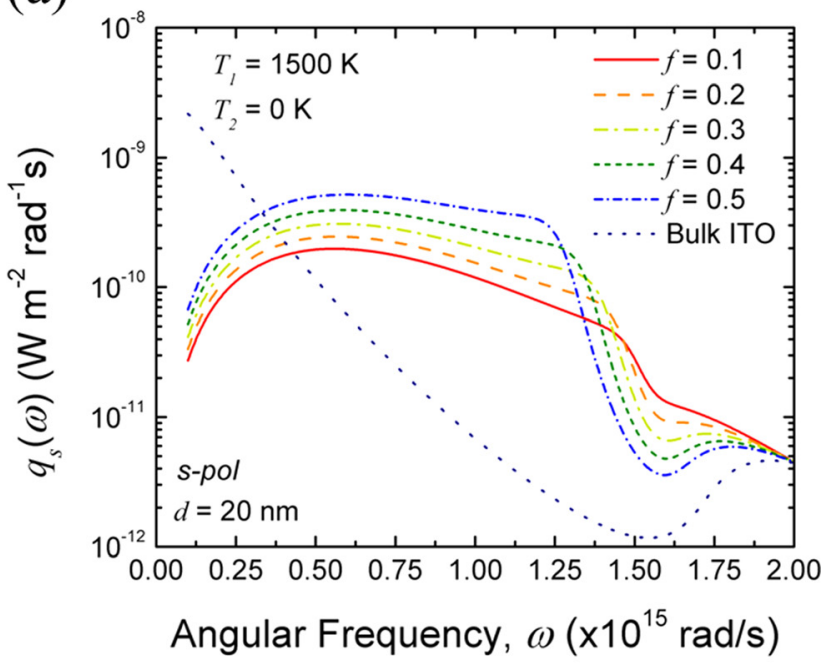

(b)

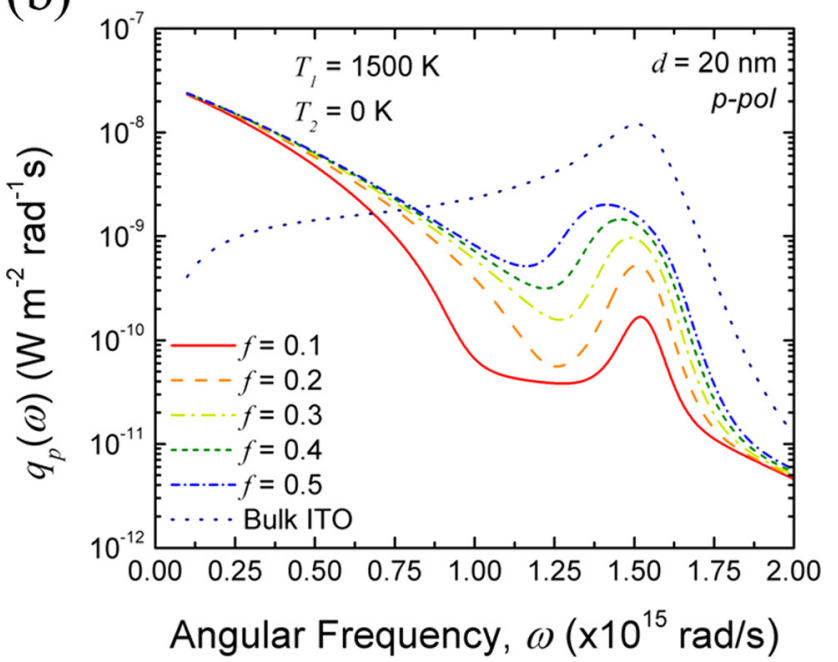

FIG. 6. Spectral heat flux of (a) s polarized and (b) p polarized waves between ITONW arrays at different symmetric filling fractions separated by $20 \mathrm{~nm}$ gap.

mode does not shift with different gap distance but will have lower amplitude at larger vacuum gap as comparing Fig. 7(b) with Fig. 6(b). However, unlike type 1 hyperbolic and ENP modes, the type 2 hyperbolic enhancement mode vanishes at $200 \mathrm{~nm}$ gap distance. This is the main reason that causes the change of trend in Fig. 5 at vacuum gap larger than $150 \mathrm{~nm}$. Figures 6 and 7 therefore illustrate the relationship between vacuum gap and hyperbolic modes for different filling fractions, which reflects in how the near-field heat transfer varies over vacuum gap for nanowires.

\section{CONCLUSION}

In summary, we have calculated the near-field heat transfer between ITO nanowires at different filling fractions using spatial dispersion dependent effective medium theory coupled with fluctuation electrodynamics. The different type of hyperbolic mode contributions at different filling fractions have been clearly illustrated in this study. Interestingly, there exists a critical vacuum gap $(150 \mathrm{~nm})$ below which the heat transfer enhancement between nanowires over bulk ITOs (a)

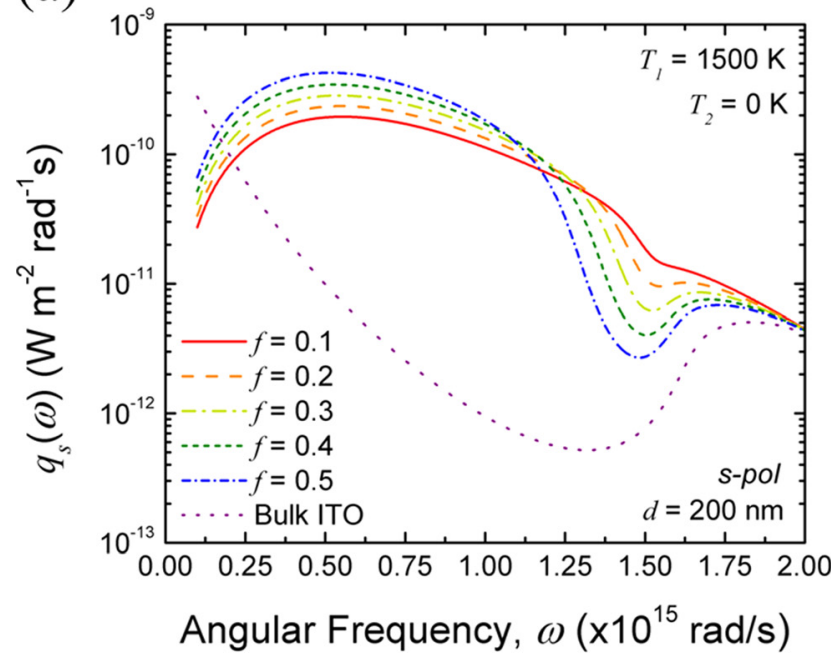

(b)

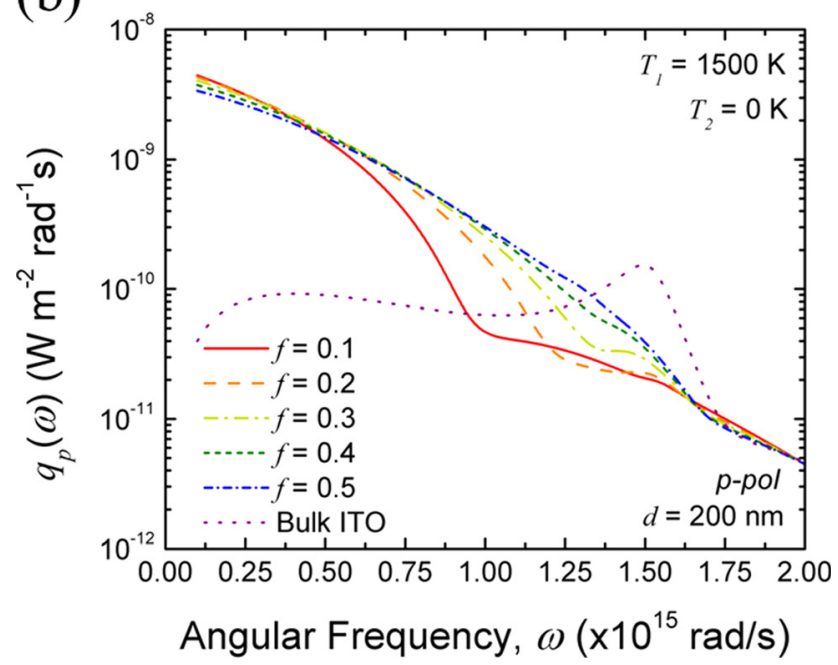

FIG. 7. Spectral heat flux of (a) s polarized and (b) p polarized waves between ITONW arrays at different symmetric filling fractions separated by $200 \mathrm{~nm}$ gap.

increases as filling fraction decreases. For distances greater than the critical gap, the trend of total heat transfer changes due to the drastic drop of type 2 hyperbolic enhancement. Results obtained from this study will facilitate the application of nanowires in optoelectronics and energy conversion systems.

\section{ACKNOWLEDGMENTS}

JYC and LW are grateful to the support from the ASU New Faculty Startup fund.

${ }^{1}$ S. Basu, Z. M. Zhang, and C. J. Fu, Int. J. Energy Res. 33, 1203 (2009).

${ }^{2}$ K. Park, S. Basu, W. P. King, and Z. M. Zhang, J. Quant. Spectrosc. Radiat. Transfer 109, 305 (2008).

${ }^{3}$ M. Laroche, R. Carminati, and J.-J. Greffet, J. Appl. Phys. 100, 063704 (2006).

${ }^{4}$ O. Ilic, M. Jablan, J. D. Joannopoulos, I. Celanovic, and M. Soljacic, Opt. Express 20, A366-A384 (2012).

${ }^{5}$ C. R. Otey, W. T. Lau, and S. Fan, Phys. Rev. Lett. 104, 154301 (2010).

${ }^{6}$ S. Basu and M. Francoeur, Appl. Phys. Lett. 98, 113106 (2011).

${ }^{7}$ Y. Yang, S. Basu, and L. Wang, Appl. Phys. Lett. 103, 163101 (2013). 
${ }^{8}$ B. Guha, C. R. Otey, C. B. Poitras, S. Fan, and M. Lipson, Nano Lett. 12, 4546 (2012).

${ }^{9}$ K. Joulain, J. Drevillon, and P. Ben-Abdallah, Phys. Rev. B 81, 165119 (2010).

${ }^{10}$ S.-A. Biehs, F. S. S. Rosa, and P. Ben-Abdallah, Appl. Phys. Lett. 98 , 243102 (2011).

${ }^{11}$ S. Basu and L. Wang, Appl. Phys. Lett. 102, 053101 (2013).

${ }^{12}$ S. Basu and M. Francoeur, Opt. Lett. 39, 1266 (2014).

${ }^{13}$ X. L. Liu and Z. M. Zhang, Appl. Phys. Lett. 104, 251911 (2014).

${ }^{14}$ Z. Jacob, MRS Bull. 37, 761-767 (2012).

${ }^{15}$ C. L. Cortes, W. Newman, S. Molesky, and Z. Jacob, J. Opt. 14, 063001 (2012).

${ }^{16}$ C. Simovski, S. Maslovski, I. Nefedov, and S. Tretyakov, Opt. Express 21, 14988-15013 (2013).

${ }^{17}$ Z. Jacob, L. V. Alekseyev, and E. Narimanov, J. Opt. Soc. Am. A 24, A52-A59 (2007).

${ }^{18}$ Y. Guo, C. L. Cortes, S. Molesky, and Z. Jacob, Appl. Phys. Lett. 101, 131106 (2012).

${ }^{19}$ Y. Guo, W. Newman, C. L. Cortes, and Z. Jacob, Adv. Optelectron. 2012, $1-9$.

${ }^{20}$ B. Liu and S. Shen, Phys. Rev. B 87, 115403 (2013).

${ }^{21}$ X. L. Liu, R. Z. Zhang, and Z. M. Zhang, Appl. Phys. Lett. 103, 213102 (2013).
${ }^{22}$ B. Liu, J. Shi, K. Liew, and S. Shen, Opt. Commun. 314, 57-65 (2014).

${ }^{23}$ E. Narimanov, M. A. Noginov, H. Li, and Y. Barnakov, in Quantum Electronics and Laser Science Conference, OSA Technical Digest (CD) (Optical Society of America, 2010), Paper No. QPDA6.

${ }^{24}$ P. R. West, S. Ishii, G. V. Naik, N. K. Emani, V. M. Shalaev, and A. Boltasseva, Laser Photonics Rev. 4, 795 (2010).

${ }^{25}$ C. Rhodes, M. Cerruti, A. Efremenko, M. Losego, D. E. Aspnes, J.-P. Maria, and S. Franzen, J. Appl. Phys. 103, 093108 (2008).

${ }^{26}$ S. Q. Li, P. Guo, L. Zhang, W. Zhou, T. W. Odom, T. Seiderman, J. B. Ketterson, and R. P. H. Chang, ACS Nano 5, 9161 (2011).

${ }^{27}$ Q. Wan, E. N. Dattoli, W. Y. Fung, W. Guo, Y. Chen, X. Pan, and W. Lu, Nano Lett. 6, 2909 (2006).

${ }^{28}$ P. A. Belov, R. Marqués, S. I. Maslovski, I. S. Nefedov, M. Silveirinha, C. R. Simovski, and S. A. Tretyakov, Phys. Rev. B 67, 113103 (2003).

${ }^{29}$ M. S. Mirmoosa, F. Rüting, I. S. Nefedov, and C. R. Simovski, J. Appl. Phys. 115, 234905 (2014).

${ }^{30}$ M. G. Silveirinha, Phys. Rev. E 73, 046612 (2006).

${ }^{31}$ T. C. Choy, Effective Medium Theory: Principles and Applications (Oxford University Press, UK, 1999).

${ }^{32}$ K. Park and Z. M. Zhang, Front. Heat Mass Transfer 4, 013001 (2013).

${ }^{33}$ S. Molesky, C. J. Dewalt, and Z. Jacob, Opt. Express 21, A96-A110 (2013). 